\title{
Otimização de um pórtico tridimensional de concreto
} armado

\author{
Halina dos Santos Salles, Paulo Frederico Souza Lomeu, Franciane Conceição \\ Peters \& Webe João Mansur
}

Universidade Federal do Rio de Janeiro, Instituto Alberto Luiz Coimbra de Pós-Graduação e Pesquisa de Engenharia (COPPE), Programa de Engenharia Civil (PEC), Laboratório de Métodos de Modelagem e Geofísica Computacional (LAMEMO), Av. Pedro Calmon, Ilha do Fundão 21941-596, Rio de Janeiro, Brasil. E-mail: salleshalina@gmail.com, paulo.lomeu@outlook.com, fran@coc.ufrj.br, webe@coc.ufrj.br

Salles H.S., Lomeu P.F.S., Peters F.C. \& Mansur W.J. (2018) Otimização de um pórtico tridimensional de concreto armado. Pesquisa e Ensino em Ciências Exatas e da Natureza, 2(edição especial): 06-14. http://dx.doi.org/10.29215/pecen.v2i2.1036

Editora acadêmica: Claudia Mazza Dias. Recebido: 30 Abril 2018. Aceito: 19 Setembro 2018. Publicado: 26 Novembro 2018.

Resumo: No projeto de edificações, os projetistas buscam soluções otimizadas e seguras relativas a diversos aspectos estruturais, além da redução dos custos de material e mão-de-obra. Os métodos de otimização podem ser empregados nesse objetivo sem comprometer a segurança estrutural. Dessa forma, este artigo tem por objetivo a otimização estrutural por Algoritmos Genéticos de um pórtico espacial de concreto armado. Para o estudo foi desenvolvido um programa de Algoritmos Genéticos, contemplando o dimensionamento das armaduras dos elementos estruturais. A função objetivo é formada pelos custos de concreto, forma e aço e será minimizada, sujeita a restrições da NBR 6118:2014 (Projeto de Estruturas de Concreto - Procedimento). O modelo estrutural é processado no programa Ansys ${ }^{\circledR}$, onde a discretização se dá via método dos elementos finitos, para a análise e obtenção dos esforços e deformações na estrutura. Neste estudo são consideradas variáveis de projeto: altura da seção transversal das vigas e dos pilares. Os resultados obtidos demostram que a metodologia forneceu respostas coerentes com a prática e, ao apresentar diferentes soluções com quase o mesmo custo, surpreenderam com soluções que poderiam ser rejeitadas por projetistas.

Palavras chave: Otimização Estrutural, Engenharia Civil, Projeto Estrutural, Análise Estrutural.

\section{Optimization of Reinforced Concrete 3D Frames}

Abstract: In building design, the designers aim a better and safer project, regarding several structural aspects, besides the reduction of the costs of the inputs. Optimization methods can be used for this purpose without compromising structural safety. Thus, this article aims at the structural optimization by genetic algorithms of reinforced concrete 3D frames. In this study a Genetic Algorithm was developed, containing the specifications for the dimensioning of the structural elements reinforcement. The objective function is formed by the costs of concrete, molds and steel and will be minimized, according to the restrictions of NBR 6118:2014 (Concrete Structures Project - Procedure). The structural model is processed in the Ansys ${ }^{\circledR}$ software, where discretization occurs through the finite element method, to analyze and obtain the stresses and deformations of the structure. The study consideres design variables: height of beams and columns. The results show that this methodology provided responses consistent when compared to the practice and also surprised with solutions that could be rejected by designers.

Key words: Structural Optimization, Civil Engineering, Structural Design, Structural Analysis.

\section{Introdução}

$\mathrm{Na}$ engenharia estrutural, alcançar equilíbrio entre economia e segurança é um desafio, visto que a diminuição considerável de insumos não pode comprometer a segurança e o comportamento da estrutura. Nesse contexto, a otimização se insere como um mecanismo que 
auxilia os projetistas a obter projeto com menor custo, garantindo a integridade e desempenho estrutural.

São muitos os trabalhos que abordam otimização de estruturas. Argolo (2000) apresentou o dimensionamento ótimo de seções retangulares de concreto armado à Flexão Composta Normal (FCN) empregando Algoritmos Genéticos (AGs). Os resultados do programa foram comparados com outros trabalhos da literatura, obtendo uma economia de até $12 \% \mathrm{em}$ relação aos outros autores.

Melo et al. (2001) formularam um problema de otimização de pórticos planos de concreto armado. A função objetivo é formada pelos custos dos insumos (concreto, aço e forma), onde as variáveis de projeto são a altura da seção transversal e as armaduras. As restrições estão associadas aos deslocamentos, a resistência e limites das variáveis de projeto.

Silva (2002) estudou Algoritmos Genéticos aplicados a dois exemplos de estruturas de concreto armado: Um trecho de pilar dimensionado à Flexão Composta Oblíqua (FCO) e um pórtico plano de cinco pavimentos. $\mathrm{O}$ autor observou que a otimização do custo do pilar não foi eficiente, devido ao fato de os indivíduos que violaram restrições terem sido eliminados da população. Para o pórtico o autor projetou uma solução através do programa Eberick ®, obtendo uma economia de $22.7 \%$ no resultado obtido pelo Algoritmo Genético, em relação à solução obtida com o programa comercial, confirmando a eficiência do método empregado.

Torres (2001) desenvolveu um procedimento para alcançar projetos ótimos de pórticos planos de concreto armado utilizando o programa Ansys ${ }^{\circledR}$. A estratégia adotada é baseada nos trabalhos Balling \& Yao (1997), seguindo os critérios da norma americana ACI (1998) para o dimensionamento. $\mathrm{O}$ autor concluiu que, na maioria dos casos, quanto maior a quantidade de variáveis de projeto maior a redução da função objetivo, porém com a otimização disponível no Ansys $\circledR^{\circledR}$ nem sempre foi observado esse comportamento.

Bastos (2004) utilizou Algoritmos Genéticos para desenvolver um programa de otimização de seções retangulares de concreto armado submetidas à FCO. O programa desenvolvido pelo autor apresentou uma economia de até $34 \%$ em relação aos resultados extraídos da literatura utilizando outros métodos de otimização.

Chaves \& Debs (2008) aplicam métodos matemáticos de otimização para obtenção do menor custo total de seções transversais de pilares de concreto armado e determinação do índice de confiabilidade. Maia (2009) utilizou o método Simplex e o Gradiente Reduzido Generalizado para minimizar o custo e seções transversais de dois projetos: uma viga em balanço com seção contínua e outra com seção variada.

Ponte (2015) aplicou técnicas de otimização do programa Ansys ${ }^{\circledR}$, com o objetivo de estudar o comportamento de uma edificação de concreto armado com 47 metros de altura e 15 pavimentos, considerando casos de cargas diferentes. A função objetivo possui apenas o propósito de reduzir o volume da seção transversal. Dessa forma, o autor conseguiu modificar e aprimorar o desempenho estrutural do edifício.

De maneira geral, os trabalhos estudaram individualmente as situações de FCN e FCO, sendo que no projeto estrutural os elementos atuam de forma solidária, isto é, como um único conjunto estrutural. Assim, para abranger o estudo de otimização em estruturas de concreto armado, este trabalho tem como objetivo a otimização por AGs de um pórtico espacial de concreto armado. Para isso, foi desenvolvido um programa de AG, contendo o dimensionamento dos elementos estruturais. A função objetivo a ser minimizada é composta pelo custo do concreto, da forma e do aço. A penalização é aplicada nos custos quando as restrições da NBR 6118:2014 (Projeto de Estruturas de Concreto - Procedimento) são violadas. As variáveis de otimização são: a altura das vigas e dos pilares. O modelo estrutural é concebido no programa Ansys ${ }^{\circledR}$, com discretização via Método dos Elementos Finitos, para a análise e obtenção dos esforços e deformações na estrutura.

\section{Metodologia}


Neste trabalho foi desenvolvido um programa de otimização utilizando AGs (Figura 1). A análise de estruturas, passo intermediário para avaliação dos indivíduos, foi realizada no programa comercial Ansys ${ }^{\circledR}$ Mechanical APDL disponível na versão estudante 18.2 pela Ansys Inc. $\mathrm{O}$ AG inicializa a população e segue para a avaliação dos indivíduos da população, criando o arquivo de entrada para o Ansys ${ }^{\circledR}$. Assim, após o AG definir os dados de entrada, parte-se para a construção do modelo computacional e a análise da estrutura via MEF no Ansys ®, que fornecerá os relatórios dos esforços internos e deformações dos elementos estruturais. O Ansys ${ }^{\circledR}$ produz dois relatórios, um para o Estado Limite de Último (ELU) e um para Estado Limite de Serviço (ELS). Em seguida, o AG faz a leitura dos relatórios gerados pelo Ansys ${ }^{\circledR}$ e prossegue para as rotinas de dimensionamento.

Assim, após a avaliação da população, o AG colocará os indivíduos em ordem (ranking) de acordo com a aptidão e então irá repetir as seguintes etapas, até o critério de parada ser satisfeito: selecionar os indivíduos para reprodução, aplicar os operadores genéticos de cruzamento e mutação, avaliar os indivíduos gerados e selecionar o que sobreviverá na população. Nessa última etapa, se o indivíduo gerado for melhor que o pior elemento da população atual, então este indivíduo será inserido na população, de acordo com sua classificação.

\section{Parâmetros de projeto}

As constantes de projeto são: geometria retangular das seções transversais, largura da seção transversal $(b)$, sobrecarga, propriedades dos materiais (módulo de elasticidade do concreto e do aço, resistência característica à compressão do concreto $\left(f_{c k}\right)$, coeficiente de Poisson $(v)$ e resistência ao escoamento do aço de armadura passiva $\left(f_{y}\right)$, cobrimento $(c)$ e os custos dos materiais e mão-de-obra.

As variáveis de projeto são a altura da seção das vigas $\left(h_{v}\right)$ e altura da seção dos pilares $\left(h_{p}\right)$. As restrições impostas para o problema são: equilíbrio das seções transversais, taxas de armadura máximas e mínimas, espaçamentos máximos e mínimos da armadura, verificação de abertura de fissuras e deformações excessivas.

\section{Função objetivo}

A função objetivo do problema de otimização a ser minimizada é o custo total do pórtico, que consiste nos custos do material e da mão-de-obra do concreto, da armadura e da forma. O custo total do pórtico é dado por $C_{T}=C_{V}+C_{P}$, onde $C_{V}$ é o custo total de todas as vigas e $C_{P}$ é o custo total de todos os pilares. Os custos das vigas e pilares, já penalizados se for o caso, serão dados por:

$$
\begin{aligned}
C_{V} & =\sum_{i=1}^{m}\left[\prod_{r=1}^{k} \operatorname{Pen}_{r}\left(C_{V_{c_{i}}}+C_{V_{a_{i}}}+C_{V_{f_{i}}}\right)\right] \\
C_{P} & =\sum_{j=1}^{n}\left[\prod_{s=1}^{p} \operatorname{Pen}_{s}\left(C_{P_{c_{j}}}+C_{P_{a_{j}}}+C_{P_{f_{j}}}\right)\right]
\end{aligned}
$$

onde $m$ é a quantidade de vigas, $C_{V_{c_{i}}}$, $C_{V_{a_{i}}}$ e $C_{V_{f_{i}}}$ é o custo referente ao concreto, aço e forma de cada viga, respectivamente, $P e n_{r}$ é a função de penalização para a $r$-ésima restrição, $k$ é a quantidade de penalizações, $n$ é a quantidade de pilares, $C_{P_{c_{j}}}$, $C_{P_{a_{j}}}$ e $C_{P_{f_{j}}}$ são o custo referente ao concreto, aço e forma de cada pilar, respectivamente, $P e n_{s}$ é a função de penalização para a sésima restrição e $p$ é a quantidade de penalizações. 


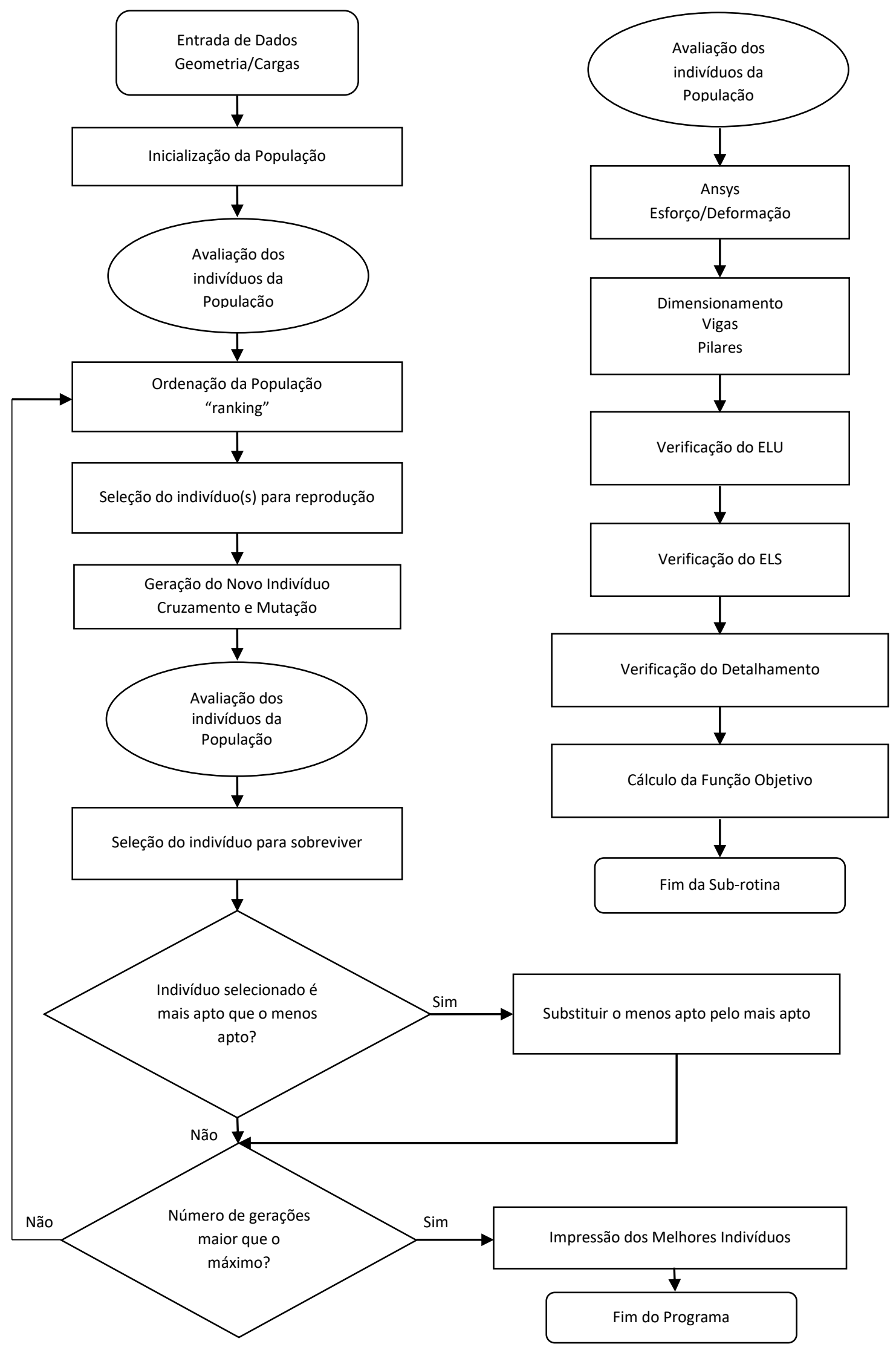

Figura 1. Fluxograma do programa desenvolvido, com detalhe à direita para a avaliação dos indivíduos. 
As Equações 3, 4 e 5 mostram as parcelas dos custos referentes às vigas.

$$
\begin{aligned}
& C_{V_{c_{i}}}=C_{c} L_{v} b h_{v} \\
& C_{V_{a_{i}}}=C_{a} y_{a} L_{l} * N b+C_{a} y_{a} L_{w}\left(L_{v} / s\right) \\
& C_{V_{f_{i}}}=C_{f} L_{v}\left(b+2 h_{v}\right)
\end{aligned}
$$

As Equações 6, 7 e 8 mostram as parcelas dos custos referentes aos pilares.

$$
\begin{aligned}
& C_{P_{c_{j}}}=C_{c} L_{p} b h_{p} \\
& C_{P_{a_{j}}}=C_{a} y_{a} L_{l} * N b+C_{a} y_{a} L_{w}\left(L_{p} / s\right) \\
& C_{P_{f_{j}}}=C_{f} L_{p} 2\left(b+h_{p}\right)
\end{aligned}
$$

Nas equações acima, $C_{c}$ é o custo do concreto por volume, $C_{a}$ é o custo do aço por peso, $C_{f}$ é o custo da forma por área, $y_{a}$ é o peso do aço, $L_{v}$ é o comprimento da viga, $b$ é a espessura da seção transversal, $L_{P}$ é o comprimento do pilar, $h_{v}$ é a altura da seção transversal da viga, $h_{p}$ é a altura da seção transversal do pilar, $L_{l}$ é o comprimento da armadura longitudinal, $L_{w}$ é o comprimento da armadura transversal, $N b$ é o número de barras longitudinais e $S$ é o espaçamento entre as armaduras transversais.

Não foram consideradas as armaduras de montagem e suplementares, somente as armaduras principais longitudinal e transversal. A ancoragem das armaduras é realizada com o comprimento de ancoragem básico, conforme a NBR 6118:2014).

\section{Critério de Parada}

Por simplicidade, uma vez que o problema a ser resolvido é relativamente simples com apenas dois parâmetros, o critério de parada adotado está relacionado ao número total de indivíduos avaliados. Assim, nos resultados aqui apresentados, o número total de soluções candidatas testadas ao longo do processo foi escolhido de tal forma que fosse maior ou igual ao número de soluções no espaço de busca, o que não seria possível realizar se o número de incógnitas fosse significativamente maior.

\section{Aplicação Numérica}

Para esse estudo será considerado um pórtico espacial (Figura 2), formado por 5 andares ( 1 térreo + 4 pavimentos), com 17.50 metros de altura e uma área em planta de 15.00 metros de extensão por 10.00 metros de largura, sendo os vãos de 5.00 metros e pé direito de 3.50 metros.

Os carregamentos considerados foram o peso próprio da estrutura $(P P)$ e sobrecarga $(S D)$ de $15 \mathrm{kN} / \mathrm{m}$. A resistência característica à compressão do concreto $\left(f_{c k}\right)$ de $30 \mathrm{MPa}$, módulo de deformação secante do concreto $\left(E_{c s}\right)$ de $27 \mathrm{GPa}$, massa específica do concreto igual a 2500 $\mathrm{kg} / \mathrm{m}^{3}$, resistência ao escoamento do aço de armadura passiva $\left(f_{y}\right)$ de $500 \mathrm{MPa}$, módulo de elasticidade do aço $\left(E_{s}\right)$ igual a $210 \mathrm{GPa}$, cobrimento $(c)$ de $30 \mathrm{~mm}$ e coeficiente de Poisson $(v)$ igual a 0.2 .

No AG as variáveis são reais, transformadas em números inteiros, uma vez que envolvem dimensões e deve-se considerar a realidade da prática da construção. O mínimo para altura dos elementos é de $25 \mathrm{~cm}$ e o máximo $50 \mathrm{~cm}$. Foram realizados três experimentos com probabilidade de crossover de $80 \%$ e taxa de mutação de $20 \%$, sendo o número de gerações o critério de parada. Para cada experimento, foram feitas 4 rodadas independentes do AG. 
No programa Ansys ${ }^{\circledR}$ foram utilizados elementos finitos de barras para a modelagem das vigas e pilares, através do elemento tridimensional BEAM188. Este é um elemento uniaxial definido internamente por dois nós de seis graus de liberdade em cada um (translações e rotações em $x, y$ e $z$ ).

Os valores utilizados para os preços de concreto, forma e aço foram extraídos do Sistema Nacional de Pesquisa de Custos e Índices da Construção Civil (SINAPI), referente ao mês de julho de 2017 para a cidade do Rio de Janeiro.

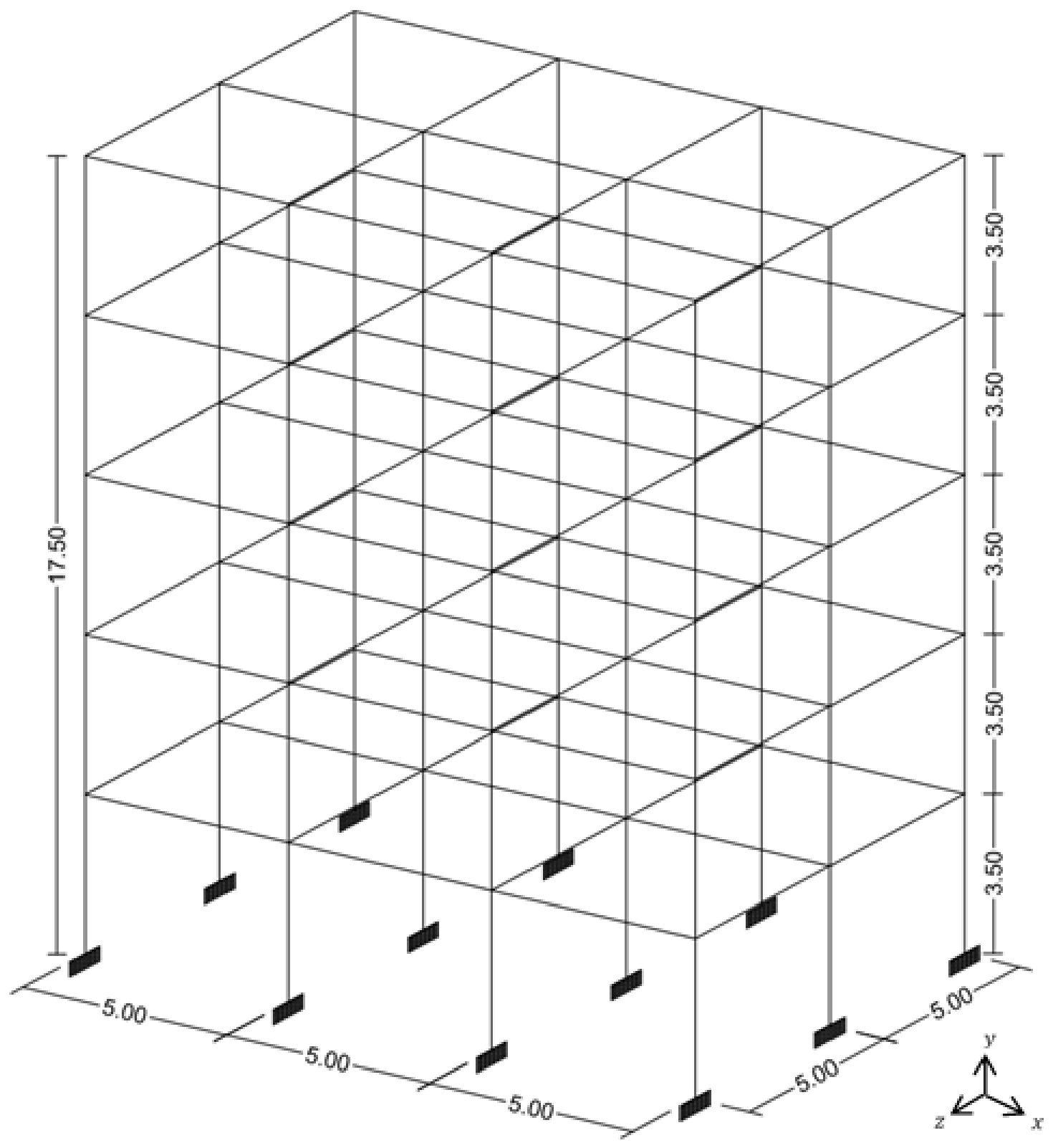

Figura 2. Pórtico espacial que terá seus elementos estruturais otimizados.

\section{Resultados e Discussão}

Para cada experimento realizado foram extraídos os resultados das avaliações em relação à altura da seção do pilar, altura da seção da viga e o custo do pórtico. Em todas as rodadas foram avaliadas 600 soluções candidatas. O primeiro experimento (Figura 3) apresenta os resultados obtidos das avaliações para população com 15 indivíduos, o segundo experimento (Figura 4) mostra as avalições para população com 30 indivíduos e o terceiro experimento (Figura 5) exibe as avalições para população com 60 indivíduos. 
Pórtico tridimensional de concreto
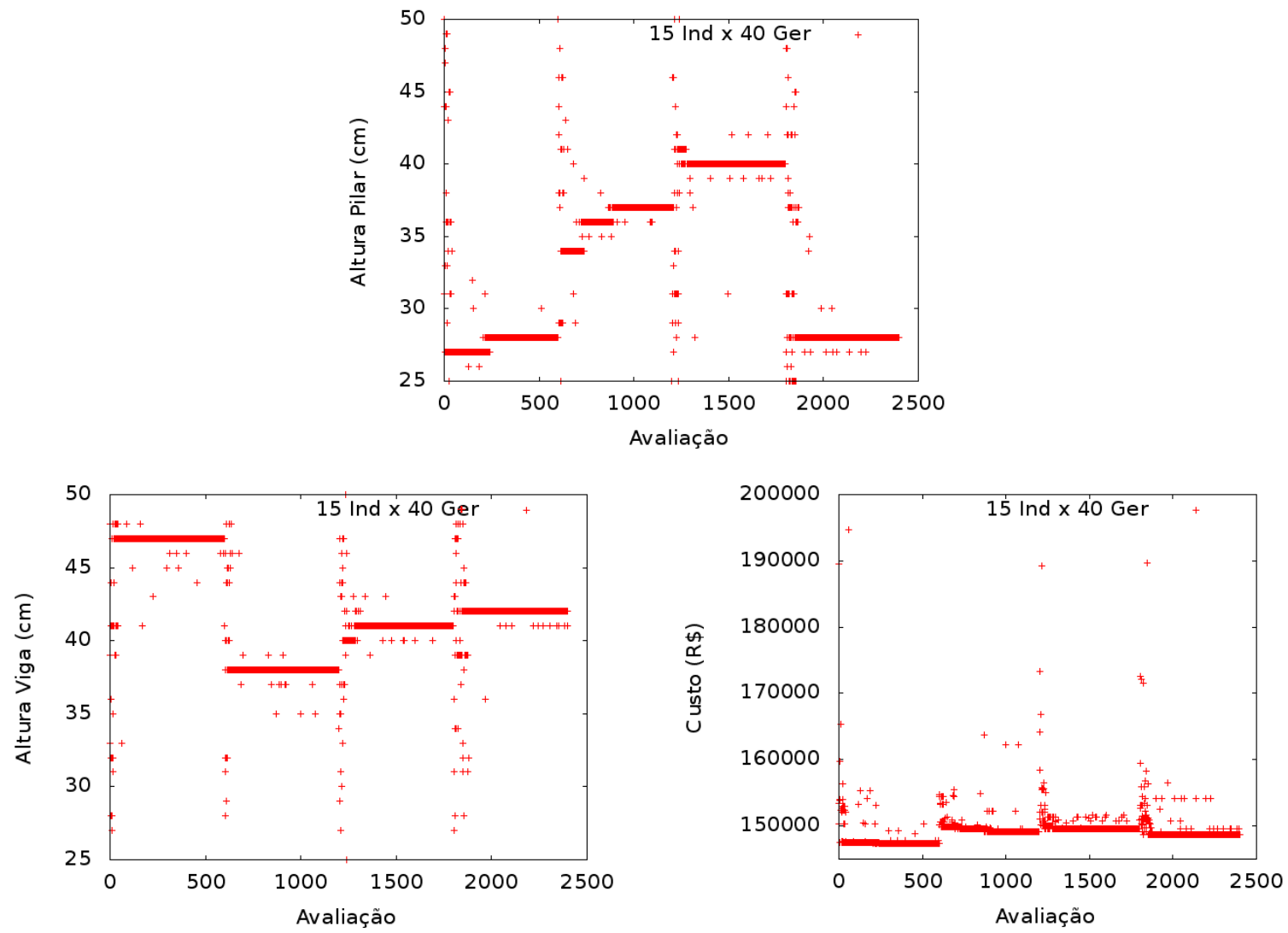

Figura 3. Avaliações para população com 15 indivíduos em 40 gerações.
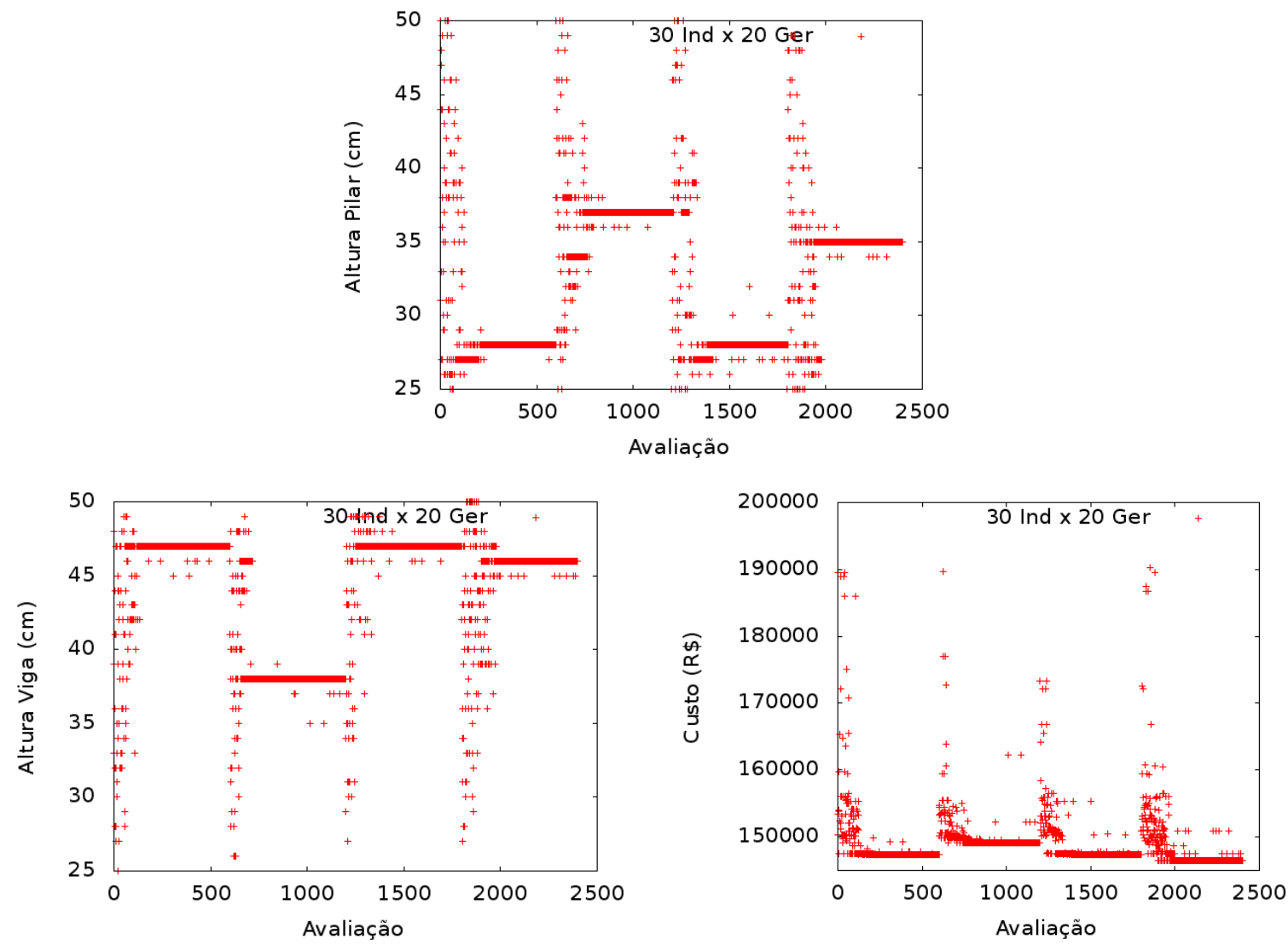

Figura 4. Avaliações para população com 30 indivíduos em 20 gerações. 

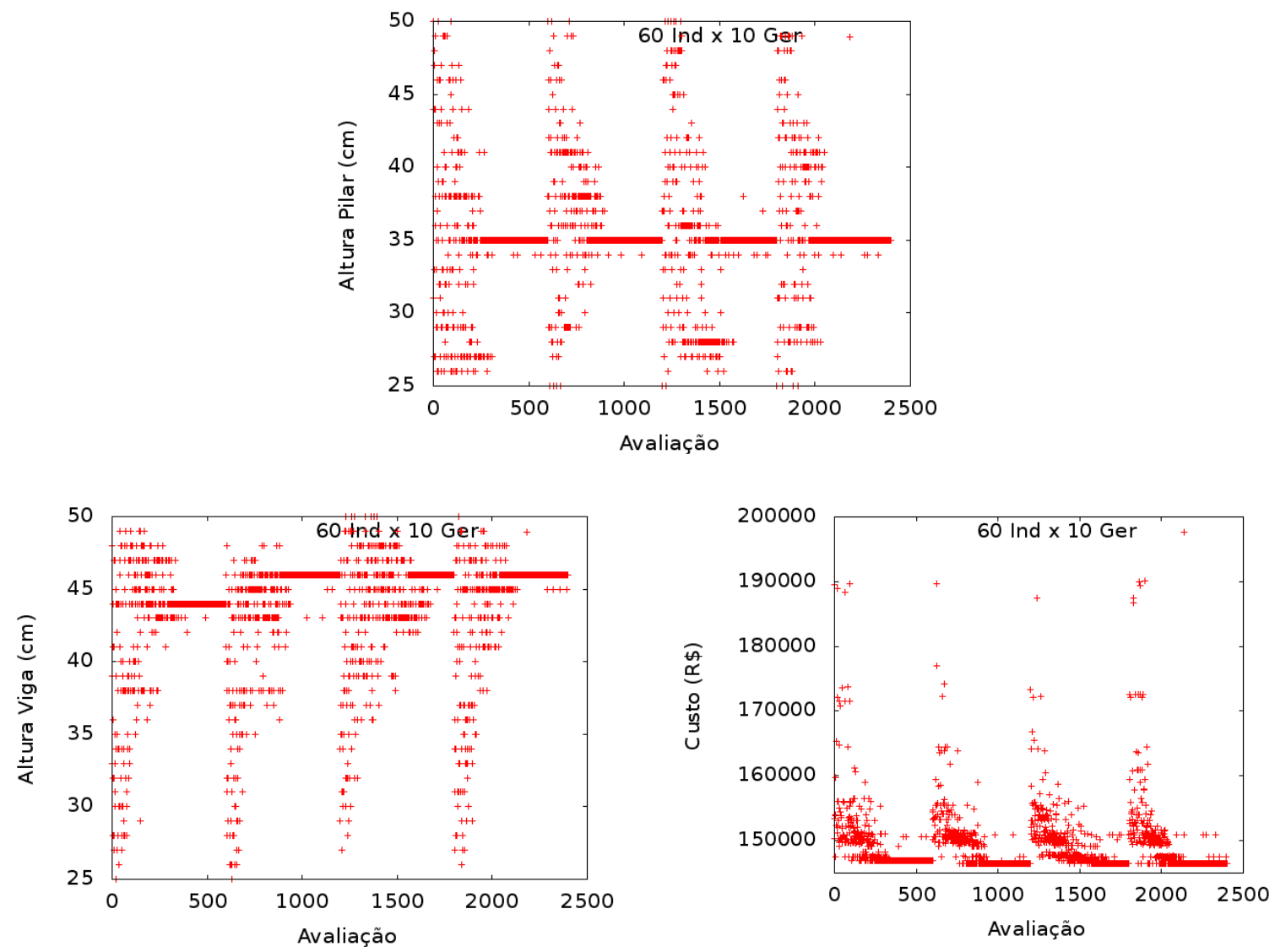

Figura 5. Avaliações para população com 60 indivíduos em 10 gerações.

No experimento com população com 15 indivíduos, foram encontradas em cada rodada 4 alturas ótimas para os pilares e vigas com custo próximo a $\mathrm{R} \$ 147.305,72$. Para 30 indivíduos o AG obteve 3 alturas ótimas para os pilares e vigas com custo de $\mathrm{R} \$ 146.468,88$. Com custo semelhante ao experimento anterior, para 60 indivíduos, os pilares atingiram $35 \mathrm{~cm}$ em todas as rodadas e as vigas chegaram a $46 \mathrm{~cm}$ em 3 rodadas.

Deve-se ressaltar que todas as soluções encontradas não violam as restrições do problema e que o aumento do número de indivíduos faz com que a solução tenda a convergir para o mínimo global do problema. Estes resultados demonstram que populações maiores aumentam a diversidade genética, e consequentemente a chance de encontrar o mínimo global.

De acordo com esses resultados é possível discutir o universo dos engenheiros para escolha da concepção estrutural de projetos. Em geral, o projeto é influenciado por vários aspectos externos, tais como a estética, funcionalidade e projetos arquitetônicos. Além disso, o engenheiro tem de buscar, entre todas as possibilidades, a estrutura mais econômica. Através dos resultados deste trabalho, o emprego de métodos de otimização se justifica pelo fato de encontrarem um conjunto de soluções, não apenas uma única solução, abrindo um leque maior de possibilidades de encontrar um melhor resultado. Os engenheiros podem comparar de maneira automatizada diversas soluções que atendam melhor os aspectos do projeto investigado, e assim elaborar sua proposta.

\section{Considerações finais}

No que diz respeito ao objetivo principal deste estudo, apresentar a otimização estrutural por Algoritmos Genéticos de um pórtico espacial de concreto armado, pode-se concluir que o mesmo foi concretizado com sucesso. Foram obtidos resultados coerentes do ponto de vista prático, além de permitir soluções, a princípio, que poderiam ser rejeitadas por projetistas. $\mathrm{O}$ 
método dos Algoritmos Genéticos apresentou um bom desempenho na otimização, funcionando como uma ferramenta que auxilia o usuário em sua tomada de decisão.

É importante destacar que o procedimento desenvolvido neste estudo auxilia no ensino de disciplinas em Engenharia Civil, tais como concreto armado, análise de estruturas, linguagem de programação, métodos numéricos e otimização. Neste trabalho foi desenvolvido um sistema que consolida vários conceitos ensinados nestas disciplinas e automatiza os procedimentos de cálculo e análise, além da interface gráfica amigável, motivando assim o estudo das mesmas.

No futuro sugere-se estudos com a variação de altura da seção dos pilares por pavimento, avaliar os parâmetros e operadores do AG, determinando-os para melhoria do desempenho, e considerar geometrias das seções transversais diferentes da retangular.

\section{Agradecimentos}

Os autores agradecem a Ansys Inc. pela disponibilização do programa de simulação para engenharia Ansys ${ }^{\circledR}$ Mechanical APDL Student e ao apoio do CNPq - Conselho Nacional de Desenvolvimento Científico e Tecnológico, da FAPERJ - Fundação Carlos Chagas Filho de Amparo à Pesquisa do Estado do Rio de Janeiro e da CAPES - Coordenação de Aperfeiçoamento de Pessoal de Nível Superior. Aos avaliadores anônimos pela revisão crítica do manuscrito.

\section{Referências}

American Concrete Institute - ACI (1998) ACI 318: Building code requirements for reinforced concrete, Farmington Hills.

Ansys Inc. (2018) Ansys ${ }^{\circledR}$ Mechanical APDL. Release 18.2. Canonsburg.

Argolo W.P. (2000) Otimização de seções de concreto armado submetidas a flexo-compressão reta utilizando Algoritmos Genéticos. Dissertação de Mestrado, COPPE, Programa de Engenharia Civil. Universidade Federal do Rio de Janeiro, Rio de Janeiro.

Associação Brasileira de Normas Técnicas - ABNT (2014) NBR 6118: Projeto de Estruturas de Concreto - Procedimento. Rio de Janeiro. 238 p.

Balling R.J. \& Yao X. (1997) How to optimize reinforced concrete frames (p. 123-137). In: Arora J.S. (Ed.). Guide to structural optimization. Volume 90. New York: The Society. 366 p.

Bastos E.A. (2004) Otimização de seções retangulares de concreto armado submetidas à flexocompressão oblíqua utilizando Algoritmos Genéticos. Dissertação de Mestrado, COPPE, Programa de Engenharia Civil. Universidade Federal do Rio de Janeiro, Rio de Janeiro.

Chaves I.A. \& Debs A.H.C. (2008) Otimização de pilares de concreto armado mediante uniformização do índice de confiabilidade. Cadernos de Engenharia de Estruturas, 10(45): 122.

Maia J.P.R. (2009) Otimização estrutural: estudo e aplicações em problemas clássicos de vigas utilizando a ferramenta solver. Dissertação de Mestrado, Escola de Engenharia de São Carlos, Programa de Pós-Graduação em Engenharia de Estruturas. Universidade de São Paulo, São Carlos.

Melo A.M.C., Vaz L.E. \& Shehata I.AE.M. (2001) Optimum design of RC plane frames with nonlinear response. $3^{\circ}$ edition ASMO UK. ISSMO Conference on Engineering Design Optimization, single volume: $165-172$.

Ponte D.S. (2015) Estudo do comportamento e otimização do projeto estrutural de edifícios de concreto armado. Dissertação de Mestrado, Faculdade de Engenharia. Universidade do Estado do Rio de Janeiro, Rio de Janeiro.

Silva E.E. (2002) Algoritmos Genéticos aplicados no dimensionamento ótimo de estruturas de concreto armado. $5^{\circ}$ Simpósio Mineiro de Mecânica Computacional, volume único: 441-448.

Torres J.V.S. (2001) Otimização de pórticos de concreto armado utilizando o sistema computacional Ansys. Dissertação de Mestrado, Departamento de Engenharia Civil. Universidade Federal de Pernambuco, Pernambuco. 